AperTO - Archivio Istituzionale Open Access dell'Università di Torino

\title{
Computational investigation of the photoinduced homolytic dissociation of water in the pyridine-water complex
}

\section{This is the author's manuscript}

Original Citation:

\section{Availability:}

This version is available http://hdl.handle.net/2318/137256

since 2016-10-06T14:14:18Z

Published version:

DOI:10.1039/c3cp44585b

Terms of use:

Open Access

Anyone can freely access the full text of works made available as "Open Access". Works made available under a Creative Commons license can be used according to the terms and conditions of said license. Use of all other works requires consent of the right holder (author or publisher) if not exempted from copyright protection by the applicable law. 


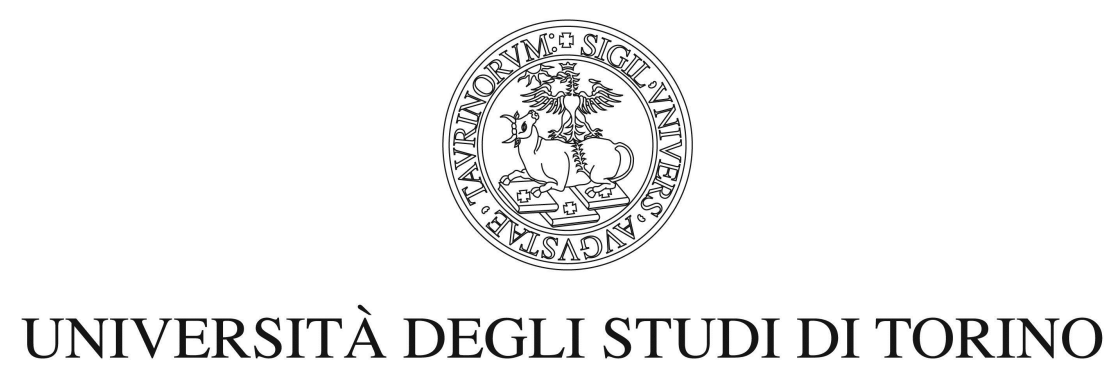

This is an author version of the contribution published on:

Xiaojun Liua, Andrzej L. Sobolewski, Raffaele Borrelli and Wolfgang Domcke. Computational investigation of the photoinduced homolytic dissociation of water in the pyridine-water complex . Physical Chemistry Chemical Physics, 15, 5957, 2013, 10.1039/C3CP44585B.

The definitive version is available at:

http://pubs.rsc.org/en/content/articlelanding/2013/cp/c3cp44585b 


\title{
Computational investigation of the photoinduced homolytic dissociation of water in the pyridine-water complex
}

\author{
Xiaojun Liu ${ }^{\mathrm{a}, \mathrm{b}^{*}}$, Andrzej L. Sobolewski ${ }^{\mathrm{c}}$, Raffaele Borrelli ${ }^{\mathrm{d}}$ and Wolfgang Domcke ${ }^{\mathrm{a}^{*}}$ \\ ${ }^{a}$ Department of Chemistry, Technical University of Munich, D-85747 Garching, Germany \\ Email:domcke@ch.tum.de \\ ${ }^{b}$ Key Laboratory of Luminescence and Optical Information, Ministry of Education, Institute of \\ Optoelectronic Technology, Beijing Jiaotong University, Beijing 100044, PR China \\ Email:xjliu@bjtu.edu.cn \\ ${ }^{c}$ Institute of Physics, Polish Academy of Sciences, PL-02668 Warsaw, Poland \\ ${ }^{d}$ Department of Agricultural, Forestry and Food Science, University of Torino, I-10095 \\ Grugliasco, Italy
}

\section{Abstract}

The photochemistry of the hydrogen-bonded pyridine-water complex has been investigated with ab initio computational methods. Vertical excitation energies, excited-state reaction paths for proton transfer as well as structures and energies of conical intersections and reaction barriers have been determined with multi-configuration self-consistent-field and multi-reference perturbation methods, as well as with single-reference coupled-cluster and propagator methods. In the pyridine-water complex, the energies of two charge-separated excited states of ${ }^{1} n \pi^{*}$ and ${ }^{1} \pi \pi^{*}$ character are connected to the energies of the locally excited ${ }^{1} \mathrm{n} \pi^{*}$ and ${ }^{1} \pi \pi^{*}$ states of the pyridine chromophore via a low energy barrier. The charge-separated excited states are strongly stabilized by the transfer of a proton from water to pyridine. The energies of the resulting biradical states intersect the potential-energy surface of the closed-shell ground state as a function of the proton-transfer coordinate. The 
resulting radical pair may dissociate to yield pyridinium and hydroxyl radicals. The photochemistry of the hypervalent pyridinium radical has been explored with the same computational methods. It has been found that a low-lying dissociative ${ }^{2} \pi \sigma^{*}$ excited state exists in pyridinium, which can lead to the photodetachment of the hydrogen atom. Overall, the $\mathrm{H}_{2} \mathrm{O}$ molecule can thus be dissociated into $\mathrm{H}^{*}$ and $\mathrm{OH}^{*}$ radicals by the absorption of two ultraviolet photons. The relevance of these results for solar water splitting and solar carbon dioxide reduction are discussed.

\section{Introduction}

Pyridine $\left(\mathrm{C}_{5} \mathrm{H}_{5} \mathrm{~N}\right.$, also abbreviated as Py in the following $)$ is a prototypical aromatic base. In aqueous solution, the solvated pyridine molecule is in equilibrium with the pyridinium cation $\left(\mathrm{C}_{5} \mathrm{H}_{5} \mathrm{NH}^{+}\right)$, the $\mathrm{pK}_{\mathrm{a}}$ value of pyridine being $5.2^{1}$. The effects of hydrogen bonding of pyridine and substituted pyridines in dilute aqueous solutions have extensively been investigated with calorimetry and spectroscopy, see, e. g., Refs. ${ }^{2-4}$ and references therein. In gas-phase Py- $\mathrm{H}_{2} \mathrm{O}$ clusters, the lone pair of the $\mathrm{N}$ atom acts as the proton acceptor of a relatively strong and nearly linear N...HO hydrogen bond. The 1:1 pyridine-water cluster was characterized by infrared (IR) spectroscopy in the gas phase as well as in rare-gas matrices ${ }^{5,6}$. The structure and the vibrational frequencies of the electronic ground state of the $\mathrm{Py}-\mathrm{H}_{2} \mathrm{O}$ complex were characterized with computational methods ${ }^{4,7-13}$. The mechanism of the protonation of pyridine in pyridine-water clusters with up to five water molecules was explored by 
Sicilia et al. with density functional theory (DFT) calculations ${ }^{14,15}$. It was shown that five water molecules are needed for the stabilization of a charge-separated $\mathrm{C}_{5} \mathrm{H}_{5} \mathrm{NH}^{+}-\mathrm{OH}^{-}$structure ${ }^{15}$.

Motivated by the conclusion of Baba et al. ${ }^{16}$ that the pyridine-water hydrogen bond may be broken in the ${ }^{1} \mathrm{n} \pi^{*}$ excited state of pyridine and the diazines, the effect of the $\mathrm{n} \rightarrow \pi^{*}$ excitation of pyridine on the hydrogen bonding in the $\mathrm{Py}-\mathrm{H}_{2} \mathrm{O}$ cluster has been investigated by Del Bene as well as by Cai and Reimers with ab initio $\operatorname{methods}^{7,12,17}$. These calculations indicate that the linear N...HO hydrogen bond prevailing in the electronic ground state is weakened in the ${ }^{1} \mathrm{n} \pi^{*}$ excited state and a hydrogen bond of the $\mathrm{H}_{2} \mathrm{O}$ molecule with the $\pi$ system of pyridine has a higher binding energy ${ }^{17}$. On the other hand, ab initio calculations of the excited-state potential-energy surfaces of the pyridine-ammonia complex with the CC2 coupled-cluster (CC) method $^{18}$ predicted the existence of ammonia-to-pyridine charge-transfer states of ${ }^{1} \mathrm{n} \pi^{*}$ and ${ }^{1} \pi \pi^{*}$ character, which are strongly stabilized in energy by the transfer of a proton from ammonia to pyridine ${ }^{19}$. The resulting hydrogen-bonded complex, consisting of the pyridinium radical $\left(\mathrm{C}_{5} \mathrm{H}_{5} \mathrm{NH}^{*}\right.$, abbreviated as $\mathrm{PyH}^{\bullet}$ in the following) and the $\mathrm{NH}_{2}{ }^{\bullet}$ radical, is lower in energy than the locally excited ${ }^{1} \mathrm{n} \pi *$ and ${ }^{1} \pi \pi^{*}$ states of the $\mathrm{Py}-\mathrm{NH}_{3}$ complex, and the activation energy for the electron/proton-transfer process is predicted to be low ${ }^{19}$. Pyridine thus acts as a photobase ${ }^{20,21}$ in the $\mathrm{Py}-\mathrm{NH}_{3}$ complex, that is, it exhibits an enhanced proton affinity in its excited states. The existence of this electron/proton-transfer process in the ${ }^{1} n \pi^{*}$ excited state of the Py- $\mathrm{H}_{2} \mathrm{O}$ cluster was recently confirmed by Reimers and Cai, using 
the equation-of-motion (EOM) CC method $^{17}$. It was shown that a hydrogen-bonded $\mathrm{PyH}^{\bullet}-\mathrm{OH}^{\bullet}$ biradical exists which is lower in energy than the locally excited states of the $\mathrm{Py}-\mathrm{H}_{2} \mathrm{O}$ complex. The activation energy of the excited-state electron/proton transfer reaction was estimated to be low, providing an explanation for the quenching of fluorescence and the observation of photohydration products of pyridine in aqueous solutions ${ }^{17}$.

The present investigation is part of our search for simple organic chromophores which potentially could serve as catalyzers for the direct photochemical homolytic splitting of water ${ }^{22-24}$. An ideal photocatalyzer, say A, should be a strong absorber in the visible range of the spectrum, should exhibit a sufficiently long excited-state lifetime and should be able to abstract a hydrogen atom from a water molecule according to the reactions

$\mathrm{A}+h v \rightarrow \mathrm{A}^{*}$

$\mathrm{A}^{*}+\mathrm{H}_{2} \mathrm{O} \rightarrow \mathrm{AH}^{*}+\mathrm{OH}^{*}$

If the $\mathrm{H}^{*}$ radical could be detached from the hypervalent radical $\mathrm{AH}^{*}$ by the absorption of an additional photon

$\mathrm{AH}^{\bullet}+h v \rightarrow \mathrm{A}+\mathrm{H}^{\bullet}$

the chromophore A is recovered and a water molecule has been split into $\mathrm{H}^{\bullet}$ and $\mathrm{OH}^{*}$ radicals by the absorption of two photons.

While pyridine itself is not a suitable chromophore for solar water splitting, since it absorbs rather far in the ultraviolet (UV), the $\mathrm{Py}-\mathrm{H}_{2} \mathrm{O}$ complex and the $\mathrm{PyH}^{\bullet}$ radical may serve as simple model systems for which the mechanistic details of the crucial 
excited-state electron/proton-transfer processes can be investigated with accurate ab initio electronic-structure methods and quantum dynamics calculations. In the present work, multi-configuration self-consistent-field (MCSCF) $)^{25,26}$ and multi-reference perturbation theory (MRPT) ${ }^{27,28}$ methods have been employed for the determination of excitation energies, excited-state reaction paths and conical intersections among the relevant electronic states of the $\mathrm{Py}-\mathrm{H}_{2} \mathrm{O}$ complex as well as the $\mathrm{PyH}^{\bullet}$ radical. The results provide a qualitative mechanistic picture of the photoinduced water-splitting reaction in the $\mathrm{Py}-\mathrm{H}_{2} \mathrm{O}$ complex and of the photoinduced $\mathrm{H}$-atom detachment process in the $\mathrm{PyH}^{\circ}$ radical. In addition, the performance of two computationally less expensive single-reference electronic-structure methods has been evaluated in the present work in comparison with the MCSCF/MRPT results. Since accurate MCSCF/MRPT methods quickly become unfeasible with increasing size of aromatic chromophores as well as with increasing number of water molecules in the clusters, it is important to assess the reliability of comparatively cheap electronic-structure methods for the description of excited-state electron/proton-transfer reactions.

\section{Computational methods}

The ground-state equilibrium geometry of the $\mathrm{Py}-\mathrm{H}_{2} \mathrm{O}$ complex was determined with the second-order Møller-Plesset (MP2) method. Excitation energies, excited-state reaction paths and energy profiles were calculated with the complete-active-space self-consistent-field (CASSCF) method ${ }^{26}$ and the CASPT2 (second-order perturbation 
theory with respect to the CASSCF reference) method ${ }^{27}$. In addition to these MCSCF and MRPT methods, the approximate second-order singles-and-doubles coupled-cluster method $(\mathrm{CC} 2)^{18}$ and the second-order algebraic-diagrammatic-construction method $(\operatorname{ADC}(2))^{29}$ were employed for the calculation of excitation energies and response properties. Geometries of selected conical intersections and saddle points were determined with the state-averaged CASSCF method. The reaction path for the electron-driven proton-transfer process from the water molecule to pyridine was constructed as a relaxed scan, that is, for a fixed value of the driving coordinate (the $\mathrm{OH}$ distance of $\mathrm{H}_{2} \mathrm{O}$ involved in the hydrogen bonding with Py) all other internal coordinates were relaxed in the respective excited state. The reaction path for the photodetachment of the hydrogen atom from the $\mathrm{PyH}^{\circ}$ radical was constructed as a rigid scan, since the relaxation of the internal coordinates of pyridine is of less importance in this case.

The choice of the active space of the CASSCF calculations for the $\mathrm{Py}-\mathrm{H}_{2} \mathrm{O}$ complex is of crucial importance, since the calculations should describe the locallyexcited states of pyridine as well as the charge-transfer states (from water to pyridine) in a balanced manner. The active space for the isolated pyridine molecule was constructed by distributing 8 electrons in 7 orbitals, including the three highest $\pi$ orbitals, the three lowest $\pi^{*}$ orbitals of pyridine as well as the $\mathrm{n}$ orbital of the $\mathrm{N}$-atom of pyridine. The active space for the $\mathrm{Py}-\mathrm{H}_{2} \mathrm{O}$ complex was enlarged by distributing 10 electrons in 9 orbitals. Besides the orbitals mentioned above, the lowest $\sigma^{*}$ orbital of water and one $\mathrm{p}$ orbital of the O-atom of water was included in the active space. For 
both Py and the Py- $\mathrm{H}_{2} \mathrm{O}$ complex, two roots (the $\mathrm{S}_{0}$ state and the lowest ${ }^{1} \pi \pi^{*}$ state) were state-averaged with equal weights in the $\mathrm{A}^{\prime}$ symmetry of the $C_{\mathrm{s}}$ point group. In the $\mathrm{A}^{\prime}$ symmetry, only the lowest ${ }^{1} \mathrm{n} \pi *$ state is considered. In the CASSCF calculations for the $\mathrm{PyH}^{\bullet}$ radical, 9 electrons were distributed in 8 orbitals. They consisted of the three highest $\pi$ orbitals and the three lowest $\pi^{*}$ orbitals of Py as well as one $\sigma$ orbital and one $\sigma^{*}$ orbital of the $\mathrm{NH}$ bond. In the $\mathrm{A}^{\prime}$ symmetry, only the lowest ${ }^{2} \pi \sigma^{*}$ state was considered. In the $\mathrm{A} "$ symmetry, four states were averaged with equal weights for the calculation of the $D_{0}$ state and three excited states of the ${ }^{2} \pi \pi^{*}$ character. The CASPT2 calculations were carried out as single-state, single-reference calculations. In all CASPT2 calculations, a level shift of 0.3 au was employed ${ }^{27}$.

$\mathrm{ADC}(2)$ is a variant of propagator theory and is closely related to the $\mathrm{CC} 2$ method. The advantage of the $\mathrm{ADC}(2)$ method compared to the $\mathrm{CC} 2$ method is that the excitation energies are calculated as the eigenvalues of a Hermitian secular matrix. The ADC(2) method is therefore able to describe conical intersections among excited states physically correctly ${ }^{29-31}$. For the $\mathrm{PyH}^{\bullet}$ radical, the unrestricted versions of the $\mathrm{CC} 2$ and $\mathrm{ADC}(2)$ methods were employed. A recent investigation of the performance of the unrestricted $\mathrm{ADC}(2)$ method for a representative test set of organic radicals has shown that the unrestricted $\mathrm{ADC}(2)$ method is a reliable alternative to coupled-cluster-based approaches for the calculation of excited states of medium-size open-shell molecules ${ }^{32}$.

Dunning's correlation-consistent split-valence double- $\square$ basis set with polarization functions on all atoms $(c c-p V D Z)^{33}$ was employed in all calculations, 
with the exception of the ${ }^{2} \pi \sigma^{*}$ state of the $\mathrm{PyH}^{*}$ radical. The Rydberg character of this state requires the augmentation of the cc-pVDZ basis with diffuse basis functions. The aug-cc-pVDZ basis was therefore employed for the calculation of the ${ }^{2} \pi \sigma^{*}$ state of $\mathrm{PyH}^{\circ}$. The MP2, CC2 and $\mathrm{ADC}(2)$ calculations were carried out with the TURBOMOLE program package ${ }^{34,35}$, making use of the resolution-of-the-identity (RI) approximation $^{36}$ for the evaluation of the electron-repulsion integrals. The CASSCF and CASPT2 calculations were performed with the MOLPRO program package ${ }^{37}$.

\section{Results}

\subsection{Photochemistry of the pyridine-water complex}

\subsubsection{Structure of $\mathrm{Py}-\mathrm{H}_{2} \mathrm{O}$}

As is well known, the $\mathrm{Py}-\mathrm{H}_{2} \mathrm{O}$ hydrogen-bonded complex exhibits an approximately linear hydrogen bond, with the lone-pair orbital of pyridine acting as a proton acceptor. The lowest-energy structure of the complex optimized at the MP2 level exhibits $\mathrm{C}_{1}$ symmetry and is shown in Fig. 1a. The calculations predict a pyridine-water hydrogen bond length $\left(R_{\mathrm{NH}}\right)$ of $2.007 \AA$ in the electronic ground state, in agreement with the result of Sicilia et al. ${ }^{14}$ There exist also local minimum-energy structures of $\mathrm{C}_{\mathrm{s}}$ symmetry (the symmetry plane is the molecular plane) and $\mathrm{C}_{\mathrm{s}}{ }^{\prime}$ symmetry (the symmetry plane is perpendicular to the molecular plane), which are shown in Figs. $1 \mathrm{~b}$ and $1 \mathrm{c}$.

The energies of the equilibrium geometries of the $\mathrm{C}_{\mathrm{s}}$ and $\mathrm{C}_{\mathrm{s}}$ ' structures are almost 
identical and are merely $0.06 \mathrm{eV}$ higher than the energy of the $C_{1}$ structure (neglecting zero-point energy corrections). The energy of the complex is thus nearly independent of the orientation of the water molecule, in agreement with earlier calculations $\mathrm{s}^{4,8-10}$, ${ }^{12-14}$. Since $C_{\mathrm{s}}$ symmetry significantly facilitates the calculation of the excited states and their potential-energy functions, the $C_{\mathrm{s}}$ symmetry constraint is applied in the present calculations if not stated otherwise.

\subsubsection{Vertical excitation energies}

The vertical excitation energies of the lowest ${ }^{1} \pi \pi^{*}$ state and the lowest ${ }^{1} n \pi^{*}$ state of the $\mathrm{Py}-\mathrm{H}_{2} \mathrm{O}$ complex are given in Table 1 . The vertical excitation energies of the isolated pyridine molecule are included for comparison. Considering first pyridine, the vertical excitation energy of the ${ }^{1} \mathrm{n} \pi^{*}$ state is $5.23 \mathrm{eV}$ and the oscillator strength is 0.008 at the CASSCF level. The ${ }^{1} n \pi^{*}$ vertical excitation energy is lowered to $4.95 \mathrm{eV}$ at the CASPT2 level. The calculated vertical excitation energy of the ${ }^{1} \pi \pi^{*}$ state is $4.95 \mathrm{eV}$ and the oscillator strength is 0.011 at CASSCF level. It is lowered to $4.74 \mathrm{eV}$ at the CASPT2 level. The latter value is somewhat lower than the experimental estimate of $4.99 \mathrm{eV}^{38}$. Both the CASSCF calculation and the CASPT2 calculation predict the vertical excitation energy of the ${ }^{1} n \pi^{*}$ state slightly above the energy of the ${ }^{1} \pi \pi^{*}$ state, in disagreement with experiment and the CASSCF and CASPT2 calculations of Cai and Reimers ${ }^{38}$. The origin of the difference from the results of Cai and Reimers is the choice of the active space of the CASSCF calculations. The active 
space employed in the present calculations is optimized for the $\mathrm{Py}-\mathrm{H}_{2} \mathrm{O}$ complex rather than for the isolated Py molecule. The $\mathrm{CC} 2$ and $\mathrm{ADC}(2)$ results for the vertical excitation energies of Py are nearly identical. They are higher than the CASPT2 energies, but predict the correct vertical order of the ${ }^{1} \mathrm{n} \pi *$ and ${ }^{1} \pi \pi^{*}$ states.

For the Py- $\mathrm{H}_{2} \mathrm{O}$ complex, the vertical excitation energy of the ${ }^{1} \mathrm{n} \pi *$ state is predicted to be $0.3 \mathrm{eV}$ higher than the vertical excitation energy of the ${ }^{1} \pi \pi^{*}$ state at the CASPT2 level. The calculation of Cai and Reimers ${ }^{12}$ predicted almost degenerate ${ }^{1} \pi \pi^{*}$ and ${ }^{1} \mathrm{n} \pi^{*}$ states at the CASPT2/aug-cc-pVDZ level. While their active space was optimized for the calculation of the locally-excited states of pyridine in the $\mathrm{Py}-\mathrm{H}_{2} \mathrm{O}$ complex, the active space of the present calculations has been chosen to obtain a balanced description of the locally-excited states as well as the charge-transfer states of Py- $\mathrm{H}_{2} \mathrm{O}$. The present vertical ${ }^{1} \pi \pi^{*}$ excitation energy of $4.82 \mathrm{eV}$ (CASPT2 level) is in rather good agreement with the maximum of the experimental absorption band of pyridine in aqueous solution $(4.84 \mathrm{eV})^{39}$. The vertical ${ }^{1} \pi \pi^{*}$ excitation energies obtained with the $\mathrm{CC} 2$ and $\mathrm{ADC}(2)$ methods are again identical. Both methods predict a near degeneracy of the vertical ${ }^{1} \pi \pi^{*}$ and ${ }^{1} \mathrm{n} \pi *$ states of the Py- $\mathrm{H}_{2} \mathrm{O}$ complex.

The frontier molecular orbitals involved in the lowest ${ }^{1} \pi \pi^{*}$ and ${ }^{1} \mathrm{n} \pi^{*}$ excited states of the pyridine-water complex are displayed in Fig. 2. The ${ }^{1} n \pi^{*}\left(\mathrm{~A}^{\prime \prime}\right)$ excited state corresponds the excitation from the n-type orbital 22a' to the $\pi$-type orbital $5 \mathrm{a}^{\prime \prime}$. The ${ }^{1} \pi \pi^{*}\left(\mathrm{~A}^{\prime}\right)$ excited state is a mixture of configurations that correspond to excitations from the two highest $\pi$ orbitals to the two lowest $\pi^{*}$ orbitals $\left(4 \mathrm{a}^{\prime \prime} \rightarrow 5 \mathrm{a}\right.$ " and $3 \mathrm{a}^{\prime \prime} \rightarrow$ $6 a^{\prime \prime)}$. It can be seen that the ${ }^{1} \pi \pi^{*}$ and ${ }^{1} n \pi^{*}$ excited states are locally excited states of 
pyridine.

\subsubsection{Reaction path for the excited-state electron/proton-transfer process}

The energy profiles along the relaxed scans for the electron/proton-transfer process from water to pyridine in the $\mathrm{S}_{0},{ }^{1} \mathrm{n} \pi^{*}$, and ${ }^{1} \pi \pi^{*}$ states of the $\mathrm{Py}-\mathrm{H}_{2} \mathrm{O}$ complex are displayed in Fig. 3. The CASSCF energy profiles are shown in Fig. 3a and the CASPT2 energy profiles (calculated at CASSCF geometries) in Fig. 3b. The reaction-path coordinate $R_{\mathrm{OH}}$ is the bond length of the $\mathrm{OH}$ group of $\mathrm{H}_{2} \mathrm{O}$ involved in the hydrogen bonding with pyridine. The left part of both figures corresponds the Py- $\mathrm{H}_{2} \mathrm{O}$ hydrogen-bonded structure, the right part $\left(R_{\mathrm{OH}}>2.0 \AA\right)$ to the hydrogen-bonded $\mathrm{PyH}^{\bullet}-\mathrm{OH}^{\bullet}$ biradical structure. The energy profiles consist of two parts which are separated, for clarity, by the dashed vertical line. The left part $\left(R_{\mathrm{OH}}<\right.$ $1.3 \AA$ ) corresponds to the Franck-Condon region of the $\mathrm{Py}-\mathrm{H}_{2} \mathrm{O}$ complex. Here the $\mathrm{S}_{0}$, ${ }^{1} \pi \pi^{*}$ and ${ }^{1} \mathrm{n} \pi *$ energies were calculated along the proton-transfer reaction path optimized in the $\mathrm{S}_{0}$ state. In the right part $\left(R_{\mathrm{OH}}>1.3 \AA\right)$, the $\mathrm{S}_{0},{ }^{1} \pi \pi^{*}$ and ${ }^{1} \mathrm{n} \pi *$ energies were calculated along the reaction path optimized in the ${ }^{1} \pi \pi^{*}$ state. The discontinuity of the $\mathrm{S}_{0}$ energy profile at $R_{\mathrm{OH}}=1.3 \AA$ reflects the different geometries of the two reaction paths.

Let us first discuss the CASSCF energy profiles (Fig. 3a). In the Franck-Condon region of the $\mathrm{Py}-\mathrm{H}_{2} \mathrm{O}$ complex, the potential-energy functions of the locally-excited ${ }^{1} \pi \pi^{*}$ and ${ }^{1} \mathrm{n} \pi^{*}$ states are parallel to that of the ground state and the ${ }^{1} \mathrm{n} \pi^{*}$ energy is 
above the ${ }^{1} \pi \pi^{*}$ energy at the CASSCF level. As expected, the shifts of the equilibrium geometries of the locally excited ${ }^{1} \pi \pi^{*}$ and ${ }^{1} n \pi^{*}$ excited states with respect to the bond length $R_{\mathrm{OH}}$ of the water molecule are minor. The right part of Fig. 3a reveals, on the contrary, that two other states of ${ }^{1} \pi \pi^{*}$ and ${ }^{1} n \pi^{*}$ character (which are rather high in energy in the Franck-Condon region) are substantially stabilized in energy by the transfer of the proton from water to pyridine. The ${ }^{1} \pi \pi^{*}$ charge-transfer state is located above the ${ }^{1} n \pi^{*}$ state throughout. Both states involve the transfer of an electron from one of the $\mathrm{p}$ orbitals of $\mathrm{H}_{2} \mathrm{O}$ to the lowest $\pi^{*}$ orbital of pyridine. The re-neutralization of the electronic charge transfer by the transfer of the proton from $\mathrm{H}_{2} \mathrm{O}$ to pyridine is the reason for the pronounced stabilization of these charge-transfer states by about 1.5 $\mathrm{eV}$. The electronic ground state, on the other hand, is strongly destabilized by the transfer of the proton. It moves up in energy by about $5.0 \mathrm{eV}$. As a result, the energy of the closed-shell $S_{0}$ state crosses the energies of the ${ }^{1} \mathrm{n} \pi^{*}$ and ${ }^{1} \pi \pi^{*}$ charge-transfer states at $R_{\mathrm{OH}} \approx 1.9 \AA$ and $R_{\mathrm{OH}} \approx 2.1 \AA$, respectively, and at an energy of about $4.5 \mathrm{eV}$ above the ground-state energy minimum (see Fig. $3 a$ ). For $R_{\mathrm{OH}}>2.1 \AA$, the energies of the two open-shell electronic states are below the energy of the closed-shell $\mathrm{S}_{0}$ state. Since an electron as well as a proton have been transferred for water to pyridine, these states represent singlet-coupled $\mathrm{PyH}^{*}-\mathrm{OH}^{*}$ biradicals.

The energy profiles for proton transfer obtained at the CASPT2 level are displayed in Fig. 3b. The excitation energies of the locally-excited states as well as the charge-transfer states are lower than at the CASSCF level, but the shape of the energy profiles is rather similar. The energy profile of the ${ }^{1} \pi \pi^{*}$ charge-transfer state crosses 
the energy of the ${ }^{1} \mathrm{n} \pi *$ charge-transfer state at $R_{\mathrm{OH}} \approx 1.4 \AA$ and becomes the lowest charge-transfer state at larger $R_{\mathrm{OH}}$. The crossings of the charge-transfer states with the $\mathrm{S}_{0}$ state occur at slightly larger $R_{\mathrm{OH}}$ and somewhat lower in energy $(\approx 4.0 \mathrm{eV})$ than at the CASSCF level, see Fig. $3 b$. The ${ }^{1} \pi \pi^{*}$ biradical state becomes the lowest electronic state for $R_{\mathrm{OH}}>2.1 \AA$.

Returning to the CASSCF results (Fig. 3a), a conical intersection of the ${ }^{1} \mathrm{n} \pi^{*}$ and ${ }^{1} \pi \pi^{*}$ locally-excited states has been determined at the CASSCF level. It is located at $R_{\mathrm{OH}} \approx 0.95 \AA$ and $6.0 \mathrm{eV}$ above the ground-state energy minimum and is represented by the blue star in Fig. 3a. The molecular structure of this conical intersection is shown in Fig. 4a. The length of the hydrogen bond $\left(R_{\mathrm{NH}}\right)$ at this conical intersection is $2.184 \AA$, which is only slightly larger than the hydrogen bond length of the ground-state equilibrium geometry. In addition, a saddle point could be found at the CASSCF level which arises from the conical intersection of the locally excited ${ }^{1} \pi \pi^{*}$ state with the ${ }^{1} \mathrm{n} \pi^{*}$ and ${ }^{1} \pi \pi^{*}$ charge-transfer states. It is located at $R_{\mathrm{OH}} \approx 1.1 \AA$ and 5.5 $\mathrm{eV}$ above the ground-state energy minimum, see the green star in Fig. 3a. The molecular structure of this saddle point is displayed in Fig. $4 \mathrm{~b}$. It is of $\mathrm{C}_{1}$ symmetry and exhibits a short and strong hydrogen bond $\left(R_{\mathrm{NH}}=1.336 \AA\right)$. The single imaginary vibrational frequency of the saddle point is $2418.7 \mathrm{~cm}^{-1}$ and corresponds to the stretching mode of the $\mathrm{OH}$ bond.

The CASSCF frontier orbitals at this saddle point are displayed in Fig. 5a (orbital 26) and Fig. 5b (orbital 27). The occupation numbers of these orbitals are 1.03 and 0.98 , respectively. It can be seen that the two frontier orbitals at this saddle point are 
of mixed $n, \pi$ and $\pi^{*}$ character. The electronic wave function at the saddle point is a mixture of the ${ }^{1} \mathrm{n} \pi^{*}$ and ${ }^{1} \pi \pi^{*}$ charge-transfer states as well as the locally excited ${ }^{1} \pi \pi^{*}$ state.

The conical intersection of the ${ }^{1} \mathrm{n} \pi^{*}$ charge-transfer state with the electronic ground state (see Fig. 3a) is a symmetry-allowed intersection (a crossing of A' and A" states) in $\mathrm{C}_{\mathrm{s}}$ symmetry, while the crossing of the ${ }^{1} \pi \pi^{*}$ charge-transfer state with the $\mathrm{S}_{0}$ state is a same-symmetry intersection. When the symmetry is relaxed to $C_{1}$, the two 2-state intersections coalesce to a 3-state conical intersection, which is represented by the red star in Fig. 3a. The molecular geometry of this 3-state conical intersection is displayed in Fig. 4c. The hydrogen bond length $\left(R_{\mathrm{OH}}\right)$ of this biradical structure is $1.984 \AA$ and its energy is about $4.6 \mathrm{eV}$ above the energy minimum of the ground state at the CASSCF level.

The energy profiles of the ${ }^{1} n \pi^{*}$ and ${ }^{1} \pi \pi^{*}$ charge-transfer excited states exhibit minima as a function of $R_{\mathrm{OH}}$ which represent $\mathrm{PyH}^{*}-\mathrm{OH}^{*}$ biradicals. The frontier molecular orbitals of these biradicals are shown in Fig. 5 c, d (A' symmetry) and Fig. 5 e, f (A" symmetry). It can be seen that the unpaired electrons occupy a p orbital in the hydroxyl radical as well as a $\pi^{*}$ orbital in the $\mathrm{PyH}^{*}$ radical. The orbitals presented in Fig. 5 illustrate that the $\mathrm{Py}-\mathrm{H}_{2} \mathrm{O}$ hydrogen-transfer photochemistry involves only compact valence-type frontier orbitals, because the electron-accepting pyridine is an unsaturated system that allows the transfer of an electron into compact $\pi^{*}$ orbitals. ${ }^{19}$

The energy profiles along the electron/proton-transfer reaction path were also calculated with the $\mathrm{ADC}(2)$ method to test the performance of this comparatively 
inexpensive computational method in comparison with the CASSCF and CASPT2 multi-reference methods. The $\mathrm{ADC}(2)$ energy profiles are displayed in Fig. 6. The locally-excited ${ }^{1} n \pi^{*}$ and ${ }^{1} \pi \pi^{*}$ states are very close in energy, as discussed above. The $\mathrm{ADC}(2)$ energy profiles of the charge-transfer states are qualitatively similar to those obtained at the CASPT2 level. The ${ }^{1} \pi \pi^{*}$ charge-transfer state is located below the ${ }^{1} \mathrm{n} \pi^{*}$ charge-transfer state for $R_{\mathrm{OH}}>1.2 \AA$. The crossings of the ${ }^{1} \pi \pi^{*}$ and ${ }^{1} \mathrm{n} \pi^{*}$ charge-transfer energies with the energy of the $\mathrm{S}_{0}$ state occur at shorter $\mathrm{OH}$ bond lengths of $\approx 1.7 \AA$ and $\approx 1.9 \AA$, respectively. The energies of these state crossings are lower by about $0.5 \mathrm{eV}$ than the curve crossings found at the CASPT2 level. Nevertheless, the results obtained with $\mathrm{ADC}(2)$ method are encouraging and indicate that this comparatively simple and fast computational method may be useful for the exploration of electron/proton-transfer potential-energy functions in hydrogen-bonded complexes involving larger organic chromophores.

The electron-driven proton-transfer reaction in the $\mathrm{Py}-\mathrm{H}_{2} \mathrm{O}$ cluster has recently also been investigated by Reimers and $\mathrm{Cai}^{17}$. The minimum energy of the ${ }^{1} \mathrm{n} \pi^{*}$ biradical was estimated with the time-dependent DFT (TDDFT) method and the EOM CC method. Reimers and Cai found evidence that the electron/proton-transfer reaction in the ${ }^{1} \mathrm{n} \pi^{*}$ state of the $\mathrm{Py}-\mathrm{H}_{2} \mathrm{O}$ cluster leads exothermically to dissociation into radicals both in the gas phase as well as in aqueous solution ${ }^{17}$. The photochemistry of the locally excited ${ }^{1} \pi \pi^{*}$ state of pyridine seems more relevant than that of the ${ }^{1} n \pi^{*}$ state, since the former is the UV-absorbing state of the $\mathrm{Py}-\mathrm{H}_{2} \mathrm{O}$ cluster. The present results indicate that the locally excited states as well as the charge-separated states of 
${ }^{1} \mathrm{n} \pi^{*}$ and ${ }^{1} \pi \pi^{*}$ character are close in energy and are strongly mixed by out-of-plane vibrational modes.

The following qualitative mechanistic picture of the photochemistry of the Py- $\mathrm{H}_{2} \mathrm{O}$ complex emerges from the potential-energy functions shown in Fig. 3 and Fig. 6. Excitation of the $\mathrm{Py}-\mathrm{H}_{2} \mathrm{O}$ complex with UV light to the bright locally-excited ${ }^{1} \pi \pi^{*}$ state results in the population of not only the ${ }^{1} \pi \pi^{*}$ state, but also of the nearly dark locally-excited ${ }^{1} \mathrm{n} \pi *$ state, because these closely spaced electronic states are strongly coupled by a conical intersection in the Franck-Condon region. The minima of these locally-excited states are separated by a relatively low barrier (the CASSCF estimate of $0.39 \mathrm{eV}$ presumably is an upper limit) from the reactive charge-transfer excited states. The charge separation of the latter drives a proton-transfer process from $\mathrm{H}_{2} \mathrm{O}$ to Py. The proton transfer results in the formation of the singlet-coupled $\mathrm{PyH}^{*}-\mathrm{OH}^{*}$ biradical. Assuming that the electron-driven proton-transfer process is ultrafast, such that the resulting excess energy is not dissipated into other vibrational degrees of freedom, the available energy is sufficient to break the hydrogen bond between the pyridinium radical and the hydroxyl radical, resulting in the free $\mathrm{PyH}^{\bullet}$ and $\mathrm{OH}^{*}$ radicals.

The dissociation of the biradical may be in competition with the relaxation to the electronic ground state via the ${ }^{1} \pi \pi^{*}-\mathrm{S}_{0}$ and ${ }^{1} \mathrm{n} \pi^{*}-\mathrm{S}_{0}$ conical intersections. That is, instead of following the diabatic path leading to biradical dissociation, the wave packet may switch to the electronic ground-state surface at the conical intersections and subsequently relax to the energy minimum of the ground state. Both paths will 
lead to the quenching of the fluorescence of pyridine in water, as observed experimentally. ${ }^{40}$ A quantitative calculation of the branching ratios at the conical intersections is required for the estimation of the quantum yields, which is beyond the scope of the present work.

\subsection{Photochemistry of the pyridinium radical}

\subsubsection{Structure and excited states of pyridinium}

The equilibrium geometry of the $\mathrm{PyH}^{\cdot}$ radical in the $\mathrm{D}_{0}\left({ }^{2} \mathrm{~A}^{\prime \prime}\right)$ ground state determined at the MP2/cc-pVDZ level exhibits $C_{2 \mathrm{v}}$ symmetry. The NH bond length in the $\mathrm{D}_{0}$ state of the $\mathrm{PyH}^{\bullet}$ radical is $1.011 \AA$, about $0.08 \AA$ shorter than the $\mathrm{C}$ - $\mathrm{H}$ bonds. The hydrogen atom connects to the nitrogen atom is slightly out of the molecular plane in the equilibrium geometry obtained at CASSCF level of theory.

The CASSCF frontier molecular orbitals involved the lowest excited electronic states of the $\mathrm{PyH}^{-}$radical are displayed in Fig. 7. For consistency with the above discussion of the $\mathrm{Py}-\mathrm{H}_{2} \mathrm{O}$ complex, we employ $\mathrm{C}_{\mathrm{s}}$ symmetry labels for the orbitals and electronic states of $\mathrm{PyH}^{*}$. The $2 \mathrm{a}^{\prime \prime}$ and $3 \mathrm{a}^{\prime \prime}$ orbitals are doubly occupied orbitals of $\pi$ character, whereas the $4 \mathrm{a}^{\prime \prime}$ orbital is the singly-occupied $\pi$ orbital, which is designated as $\pi^{\#}$ orbital in the following. The $5 \mathrm{a}^{\prime \prime}$ and $6 \mathrm{a}^{\prime \prime}$ orbitals are unoccupied $\left(\pi^{*}\right)$ orbitals, while the orbital $19 \mathrm{a}^{\prime}$ is the $\sigma^{*}$ orbital of the NH bond. The lowest excited state is a dark state and corresponds to the $4 \mathrm{a}^{\prime \prime} \rightarrow 5 \mathrm{a} "$ transition. It is therefore designated as ${ }^{2} \pi^{\#} \pi^{*}$ excited state. The second lowest excited state corresponds to the excitation from 
the $4 a^{\prime \prime}$ orbital to the $19 \mathrm{a}^{\prime}$ orbital and is designated as ${ }^{2} \pi^{\#} \sigma^{*}$ excited state. The excitation from the $\pi^{\#}$ orbital to the $\sigma^{*}$ orbital involves a certain amount of charge transfer from the pyridine ring to the hydrogen atom, which is reflected by a relatively large dipole moment of the ${ }^{2} \pi^{\#} \sigma^{*}$ state (7.5 Debye). The third lowest excited state, which also is a dark state, is a mixture of the $2 \mathrm{a}^{\prime \prime} \rightarrow 4 \mathrm{a}^{\prime}$ configuration (major) and the $4 \mathrm{a}^{\prime \prime} \rightarrow 6 \mathrm{a} "$ configuration (minor). It is the lowest ${ }^{2} \pi \pi^{\#}$ state. The fourth excited state is of ${ }^{2} \pi^{\#} \pi^{*}$ character ( $3 \mathrm{a}^{\prime \prime} \rightarrow 4 \mathrm{a}^{\prime}$ excitation) and is the lowest bright state of $\mathrm{PyH}^{*}$.

The vertical excitation energies of the $\mathrm{PyH}^{\bullet}$ radical at the equilibrium geometry of the ground state are given in Table 2. The vertical excitation energies of the ${ }^{2} \pi^{\#} \pi^{*}$ excited state, the ${ }^{2} \pi^{\#} \sigma^{*}$ excited state and the two ${ }^{2} \pi \pi^{\#}$ excited states are $1.84 \mathrm{eV}, 2.11$ $\mathrm{eV}, 4.45 \mathrm{eV}$ and $5.98 \mathrm{eV}$, respectively, at the CASSCF level. The excitation energies are $1.50 \mathrm{eV}, 2.26 \mathrm{eV}, 4.16 \mathrm{eV}$ and $5.06 \mathrm{eV}$, respectively, at the CASPT2 level. The $\mathrm{ADC}(2)$ and $\mathrm{CC} 2$ methods give slightly lower excitation energies for the ${ }^{2} \pi^{\#} \sigma^{*}$ excited state and somewhat higher excitation energies for the ${ }^{2} \pi^{\#} \pi^{*}$ and ${ }^{2} \pi \pi^{\#}$ excited states. We are not aware of other computational or experimental data for the excitation energies of the pyridinium radical.

\subsubsection{Reaction path for the excited-state hydrogen-detachment process}

The PE profiles for the rigid detachment of the hydrogen atom from the $\mathrm{NH}$ group of the pyridinium radical, calculated with the CASSCF and CASPT2 methods, are shown in Fig. 8. All internal coordinates, except the NH distance, have been kept 
fixed at the equilibrium geometry of the electronic ground state of $\mathrm{PyH}^{*}$. The CASSCF profiles are shown as lines and the CASPT2 curves as lines with symbols. The energy profiles of the $D_{0}$ state, the ${ }^{2} \pi^{\#} \sigma^{*}$ excited state, the ${ }^{2} \pi^{\#} \pi^{*}$ excited state, the dark ${ }^{2} \pi \pi^{\#}$ state and the bright ${ }^{2} \pi \pi^{\#}$ excited state are shown in black, red, blue, cyan and green, respectively.

While the PE functions of the ${ }^{2} \pi^{\#} \pi^{*}$ and ${ }^{2} \pi \pi^{\#}$ excited states are parallel to that of the $D_{0}$ state, the ${ }^{2} \pi^{\#} \sigma^{*}$ state behaves quite differently. In the Franck-Condon zone of pyridinium, the ${ }^{2} \pi^{\#} \sigma^{*}$ state has the character of a Rydberg state. When the NH bond is stretched, the Rydberg-like $\sigma^{*}$ orbital collapses towards the $1 \mathrm{~s}$ orbital of the hydrogen atom. This transformation of the $\sigma^{*}$ orbital from a diffuse Rydberg orbital to a compact valence orbital is manifested by the low barrier in the ${ }^{2} \pi^{\#} \sigma^{*}$ PE profile at intermediate $\mathrm{NH}$ distances at the CASSCF level, see Fig. 8. This barrier almost disappears at CASPT2 level. The PE profile of the ${ }^{2} \pi^{\#} \sigma^{*}$ state of pyridinum clearly is reminiscent of the ${ }^{1} \pi \sigma^{*} \mathrm{PE}$ profiles of pyrrole and indole ${ }^{41}$.

The essentially dissociative PE function of the ${ }^{2} \pi^{\#} \sigma^{*}$ state crosses the PE functions of the ${ }^{2} \pi^{\#} \pi^{*}$ state and the $D_{0}$ state. These crossings are symmetry-allowed crossings in $C_{2 \mathrm{v}}$ symmetry. When symmetry-lowering vibrational modes are taken into account, these curve crossings become conical intersections. For pyrrole, indole and related aromatic chromophores with acidic groups $\left(\mathrm{NH}, \mathrm{OH}\right.$ or $\left.\mathrm{NH}_{2}\right)$, it is well documented that the ${ }^{1} \pi \sigma^{*}$ states lead to photodissociation with significant quantum yields. If the ${ }^{2} \pi^{\#} \sigma^{*}$ state of pyridinium can be populated by radiationless relaxation from the bright ${ }^{2} \pi \pi^{\#}$ excited state, it may provide a channel for efficient H-atom 
elimination. The excess energy being available after the radiationless decay of the bright ${ }^{2} \pi \pi^{\#}$ state will certainly be sufficient to overcome the low barrier in the PE function of the ${ }^{2} \pi^{\#} \sigma^{*}$ state (see Fig. 8).

The geometries and energies of the conical intersections of the ${ }^{2} \pi^{\#} \sigma^{*}$ surface with the ${ }^{2} \pi^{\#} \pi^{*}$ and $\mathrm{D}_{0}$ surfaces were determined at the CASSCF/cc-pVDZ level. The ${ }^{2} \pi^{\#} \pi^{*-}{ }^{2} \pi^{\#} \sigma^{*}$ conical intersection has $C_{2 \mathrm{v}}$ symmetry. The NH bond length of this conical intersection is $R_{\mathrm{NH}}=1.280 \AA$ at the CASSCF level. At the CASPT2 level, the $\mathrm{NH}$ bond length of this conical intersection is about $0.1 \AA$ longer. The $\mathrm{D}_{0^{-}}{ }^{2} \pi^{\#} \sigma^{*}$ conical intersection also has $C_{2 \mathrm{v}}$ symmetry. The $\mathrm{NH}$ bond length of this conical intersection is considerably stretched $\left(R_{\mathrm{NH}}=1.321 \AA\right)$. The crossing of the $\mathrm{D}_{0}$ and ${ }^{2} \pi^{\#} \sigma^{*}$ energy profiles occurs at $R_{\mathrm{NH}} \approx 1.47 \AA$ at the CASSCF and CASPT2 levels (see Fig. 8), which is somewhat larger than the CASSCF-optimized geometry of the conical intersection. This reflects the fact that the energy curves in Fig. 8 were calculated for a rigid detachment of the H-atom rather than along a relaxed path.

The energy profiles for rigid hydrogen detachment in the $\mathrm{PyH}^{\circ}$ radical were also calculated with the $\operatorname{ADC}(2)$ method to test the performance of this method. The results are given in Fig. 9. Comparing the ADC(2) energy profile of the ${ }^{2} \pi^{\#} \sigma^{*}$ state in Fig. 9 with the CASPT2 energy curve in Fig. 8, it is seen that $\operatorname{ADC}(2)$ predicts a slightly higher barrier in the ${ }^{2} \pi^{\#} \sigma^{*}$ PE function. The top of the barrier moves from $R_{\mathrm{NH}}$ $\approx 1.3 \AA$ at the CASPT2 level to $R_{\mathrm{NH}} \approx 1.5 \AA$ at the $\mathrm{ADC}(2)$ level. The crossing of the ${ }^{2} \pi^{\#} \sigma^{*}$ and the $\mathrm{D}_{0}$ energies occurs at $R_{\mathrm{NH}} \approx 1.5 \AA$ for both methods. The crossing of the ${ }^{2} \pi^{\#} \pi^{*}$ and ${ }^{2} \pi^{\#} \sigma^{*}$ excited states, on the other hand, shifts to significantly shorter NH 
distances at the ADC(2) level due to the significantly higher energy of the ${ }^{2} \pi^{\#} \pi^{*}$ state. It is likely that the CASPT2 calculation yields an upper limit for the latter crossing point, while the $\mathrm{ADC}(2)$ level provides a lower limit. While neither the CASPT2 method nor the $\operatorname{ADC}(2)$ method are sufficiently accurate for a quantitative prediction of the dissociation barriers and the energies and geometries of the electronic curve crossings, it is encouraging that the single-reference $\mathrm{ADC}(2)$ method yields results for reactive PE functions which are in qualitative agreement with the considerably more expensive multi-reference CASPT2 calculations. The ADC(2) method can thus be recommended for the qualitative exploration of excited-state PE surfaces of larger aromatic systems molecules and supramolecular complexes.

\section{Discussion and outlook}

$\mathrm{Ab}$ initio electronic-structure computational methods were employed in the present work for the exploration of fundamental mechanistic aspects of photoinduced electron/proton-transfer reactions in the hydrogen-bonded $\mathrm{Py}-\mathrm{H}_{2} \mathrm{O}$ complex. The locally-excited and the charge-separated states, their energy profiles along the proton-transfer reaction path, as well as the energy crossings (conical intersections) among themselves and with the electronic ground state have been characterized with MCSCF and MRPT methods. As indicated by previous single-reference calculations ${ }^{17}$, the charge-transfer states of the $\mathrm{Py}-\mathrm{H}_{2} \mathrm{O}$ cluster drive a proton-transfer reaction from $\mathrm{H}_{2} \mathrm{O}$ to Py, resulting in the formation of the hypervalent $\mathrm{PyH}^{\bullet}$ radical and the $\mathrm{OH}^{\bullet}$ 
radical.

The photochemistry of the $\mathrm{Py}-\mathrm{H}_{2} \mathrm{O}$ complex is an exemplar of a more general class of electron-driven proton-transfer reactions, which were previously characterized with computational methods for hydrogen-bonded complexes of aromatic chromophores with acidic groups (such as phenol or indole) with amphoteric solvent molecules (such as water or ammonia), as well as for hydrogen-bonded aromatic pairs (such as pyrrole-pyridine or guanine-cytosine) $)^{19}$. In all these systems, optically dark charge-separated excited electronic states play a decisive role in the photochemistry. The transfer of a proton of a hydrogen bond neutralizes the electronic charge separation ("the proton follows the electron"), which results in a pronounced stabilization of the resulting biradical states. The electronic ground state, on the other hand, is strongly destabilized by the proton transfer. Together, these effects give rise to generic conical intersections of the biradical states with the electronic ground state ${ }^{19}$. These conical intersections play a decisive role for the photochemistry of these hydrogen-bonded complexes. If the excited-state wave packet follows the diabatic path at the conical intersection, the complex dissociates into two radicals. If the excited-state wave packet follows the adiabatic path, on the other hand, the dissociation process is aborted and the complex relaxes to the minimum of the $\mathrm{S}_{0}$ potential-energy surface ${ }^{19}$. In the $\mathrm{Py}_{-} \mathrm{H}_{2} \mathrm{O}$ complex, the former outcome leads to homolytic water splitting. The latter outcome enhances the photostability of pyridine in an aqueous environment.

An important open question concerning the mechanism of the water-dissociation 
reaction in the photoexcited $\mathrm{Py}-\mathrm{H}_{2} \mathrm{O}$ complex is the role of triplet states. Since the two singly occupied molecular orbitals of the charge-transfer and/or biradical states are non-overlapping, the singlet-coupled and triplet-coupled excited states are essentially degenerate. Due to this degeneracy, efficient intersystem crossing may occur along the whole proton-transfer reaction path, which may result in a potentially significant population of triplet-coupled biradicals, despite the fact that the spin-orbit interaction is relatively weak. Nuclear wave packets emerging in the dissociative triplet state will cross the $\mathrm{S}_{0}$ potential-energy surface essentially freely, in contrast to wave packets in the singlet-coupled biradical states, which encounter a conical intersection with the $\mathrm{S}_{0}$ surface, as discussed above. Intersystem crossing may thus enhance the radical yield beyond the radical yield resulting in the singlet manifold.

The photochemistry of the pyridinium radical is an exemplar of the UV photodissociation of a hypervalent aromatic radical via a repulsive ${ }^{2} \pi \sigma^{*}$ excited state. This photochemical process, which seems to have received little attention so far, bears similarities with the photodissociation of aromatic chromophores with acidic groups (such as pyrrole, phenol, or aniline) via repulsive ${ }^{1} \pi \sigma^{*}$ excited states. The photochemistry of the latter systems has extensively been explored in recent years with experimental and computational methods and is therefore well understood. As discussed in Section 3, the $\mathrm{PyH}^{\bullet}$ radical can potentially be dissociated via a low-lying dissociative ${ }^{2} \pi \sigma^{*}$ state. The theoretical description of the photodissociation dynamics of hypervalent radicals is complicated, however, by the existence of low-lying non-reactive ${ }^{2} \pi \pi^{\#}$ and ${ }^{2} \pi^{\#} \pi^{*}$ excited states, which results in a larger number of 
same-spin curve crossings in the photodisociation dynamics than in closed-shell systems.

By the abstraction of a hydrogen atom from water in the $\mathrm{Py}-\mathrm{H}_{2} \mathrm{O}$ complex, a major fraction $(\approx 80 \%)$ of the excitation energy of the bright ${ }^{1} \pi \pi^{*}$ state of pyridine is converted into the chemical energy of two ground-state radicals. The energy conversion of the $\mathrm{H}$-atom photodetachment reaction from the $\mathrm{PyH}^{\bullet}$ radical is less favorable. Due to the rather high excitation energy of the bright ${ }^{2} \pi \pi^{\#}$ state and the rather low dissociation energy of the ${ }^{2} \pi \sigma^{*}$ state, only about $30 \%$ of the photon energy are stored as chemical energy in this process. It may thus be preferable to exploit the high reducing potential of the $\mathrm{PyH}^{*}$ radical directly in a follow-up chemical reaction, as discussed below.

Since the photoexcitation of pyridine to the ${ }^{1} \pi \pi^{*}$ state requires UV light $(\lambda \approx 250$ $\mathrm{nm}$ ), pyridine is not a suitable photocatalyzer for solar water splitting. However, the excitation energies of ${ }^{1} \pi \pi^{*}$ states of aromatic systems can be lowered simply by the extension of the conjugated bond system. Acridine $\left(\mathrm{C}_{13} \mathrm{H}_{9} \mathrm{~N}\right)$, for example, has a $\mathrm{pK}_{\mathrm{a}}$ of 5.6 which is similar to that of pyridine and absorbs at $\approx 360 \mathrm{~nm}$. Acridine orange $\left(\mathrm{N}, \mathrm{N}, \mathrm{N}^{\prime}, \mathrm{N}^{\prime}\right.$-tetramethylacridine-3,6-diamine) has the absorption maximum at $\approx 500$ $\mathrm{nm}$, already in the visible spectral range. In contrast to the band gap of semiconductors, the excitation energies of aromatic dyes can easily be tuned over a wide range. If the excitation energies of the water-to-chromophore charge-transfer states in such hydrogen-bonded complexes can also be lowered such that they are accessible after ${ }^{1} \pi \pi^{*}$ excitation of these chromophores, these dyes will catalyze the 
homolytic water-dissociation reaction. The energy conversion factor of the H-atom photodetachment reaction from the aromatic radical will become more favorable as well.

Alternatively to the photocleavage of $\mathrm{PyH}^{\bullet}$, the chemical energy of the radical can be harvested by the exothermic recombination of two $\mathrm{PyH}^{\bullet}$ radicals

$$
2 \mathrm{PyH}^{\bullet} \rightarrow 2 \mathrm{Py}+\mathrm{H}_{2}
$$

The $\mathrm{OH}^{\bullet}$ radicals, on the other hand, are waste products and should efficiently be scavenged and recombined to $\mathrm{H}_{2} \mathrm{O}$ and $\mathrm{O}_{2}$ by a suitable catalyzer in order to minimize the damage of the photocatalyzer by the highly reactive $\mathrm{OH}^{*}$ radicals.

The reduction potential of the $\mathrm{PyH}^{\bullet}$ radical is sufficient for the reduction of carbon dioxide to the formyl radical:

$$
\mathrm{PyH}^{\bullet}+\mathrm{CO}_{2} \rightarrow \mathrm{Py}+\mathrm{COOH}^{*}
$$

A second $\mathrm{PyH}^{\bullet}$ radical can reduce the formyl radical to formic acid:

$$
\mathrm{PyH}^{\bullet}+\mathrm{COOH}^{\bullet} \rightarrow \mathrm{Py}+\mathrm{HCOOH}
$$

Four additional $\mathrm{PyH}^{*}$ radicals can reduce formic acid to methanol:

$4 \mathrm{PyH}^{\bullet}+\mathrm{HCOOH} \rightarrow 4 \mathrm{Py}+\mathrm{CH}_{3} \mathrm{OH}+\mathrm{H}_{2} \mathrm{O}$.

The photocatalyzer pyridine is recovered by these dark reactions. The reduction of $\mathrm{CO}_{2}$ to formic acid and methanol by the pyridinium radical has recently been demonstrated in electrochemical experiments, in which $\mathrm{PyH}^{*}$ radicals were generated by the neutralization of pyridinium cations ${ }^{42,43}$.

If the $\mathrm{PyH}^{\circ}$ radical in these reactions could be replaced by an reducing agent $\mathrm{AH}^{\circ}$ which can be obtained by homolytic water splitting in an $\mathrm{A}-\mathrm{H}_{2} \mathrm{O}$ complex which 
absorbs near the maximum of the solar spectrum, carbon dioxide and water could efficiently be processed to methanol and oxygen with a single and cheap photocatalyzer.

\section{Acknowledgements}

This work was supported by the National Natural Science Foundation of China (X. L., Grant No. 21003009), China Scholarship Council, by the National Science Center of Poland (A. L. S., Grant No. N N202 126337) and by a research grant of the Deutsche Forschungsgemeinschaft (W. D.). A. L. S. and R. B. acknowledge support by the DFG cluster of excellence "Munich Centre for Advanced Photonics".

\section{References:}

1 A. Fischer, W. J. Galloway and J. Vaughan, J Chem. Soc., 1964, 3591.

2 E. M. Arnett, B. Chawla, L. Bell, M. Taagepera, W. J. Hehre and R. W. Taft, J Am. Chem. Soc., 1977, 99, 5729.

3 J. J. Valentini, M. J. Coggiola and Y. T. Lee, J Am. Chem. Soc., 1976, 98, 853.

4 S. Schlücker, R. K. Singh, B. P. Asthana, J. Popp and W. Kiefer, J. Phys. Chem. A, 2001, 105, 9983.

5 D. J. Millen and G. W. Mines, J. Chem. Soc. Faraday Trans. (2), 1977, 73, 369.

6 A. Destexhe, J. Smets, L. Adamowicz and G. Maes, J. Phys. Chem., 1994, 98, 1506.

7 J. E. Del Bene, J Am. Chem. Soc., 1975, 97, 5330.

8 M. A. Martoprawiro and G. B. Bacskay, Mol. Phys., 1995, 85, 573.

9 I. Pápai and G. Jancsó, J. Phys. Chem. A, 2000, 104, 2132.

10 A. Dkhissi, L. Adamowicz and G. Maes, J. Phys. Chem. A, 2000, 104, 2112.

11 Y. Li, R. Lu, Y. Hu and X. Wang, Chem. Phys. Lett., 2001, 333, 153.

12 Z. Cai and J. R. Reimers, J. Phys. Chem. A, 2002, 106, 8769.

13 D. Singh, S. K. Srivastava, A. K. Ojha, B. P. Asthana and R. K. Singh, J. Mol. Struct:THEOCHEM, 2007, 819, 88.

14 M. C. Sicilia, C. Muñoz-Caro and A. Niño, Chemphyschem, 2005, 6, 139. 
40 T. Handa, Y. Utena, H. Yajima, T. Ishii and H. Morita, J. Phys. Chem., 1986, 90, 2589.

41 A. L. Sobolewski, W. Domcke, C. Dedonder-Lardeux and C. Jouvet, Phys. Chem. Chem. Phys., 2002, 4, 1093.

42 E. Barton Cole, P. S. Lakkaraju, D. M. Rampulla, A. J. Morris, E. Abelev and A. B. Bocarsly, J Am. Chem. Soc., 2010, 132, 11539.

43 A. J. Morris, R. T. McGibbon and A. B. Bocarsly, ChemSusChem, 2011, 4, 191. 
Table 1. Vertical excitation energies (in $\mathrm{eV}$ ) and oscillator strengths (in parentheses) of pyridine and the pyridine-water complex.

\begin{tabular}{ccccc}
\hline & \multicolumn{2}{c}{ pyridine } & \multicolumn{2}{c}{ pyridine-water } \\
\cline { 2 - 5 } & ${ }^{1} \mathrm{n} \pi^{*}\left(\mathrm{~A}^{\prime \prime}\right)$ & ${ }^{1} \pi \pi^{*}\left(\mathrm{~A}^{\prime}\right)$ & ${ }^{1} \mathrm{n} \pi^{*}\left(\mathrm{~A}^{\prime \prime}\right)$ & ${ }^{1} \pi \pi^{*}\left(\mathrm{~A}^{\prime}\right)$ \\
\hline ADC(2) & $5.13(0.004)$ & $5.35(0.025)$ & $5.33(0.004)$ & $5.34(0.030)$ \\
$\mathrm{CC} 2$ & $5.16(0.004)$ & $5.34(0.022)$ & $5.36(0.004)$ & $5.33(0.025)$ \\
CASSCF & $5.23(0.008)$ & $4.95(0.011)$ & $5.70(0.006)$ & $5.00(0.019)$ \\
CASPT2 & 4.95 & 4.74 & 5.12 & 4.82 \\
Others & $4.93^{\mathrm{a}}$ & $4.88^{\mathrm{a}}$ & $4.96^{\mathrm{c}}$ & $4.95^{\mathrm{c}}$ \\
& $4.74^{\mathrm{b}}$ & $4.99^{\mathrm{b}}$ & & $4.84^{\mathrm{d}}$ \\
\hline
\end{tabular}

${ }^{\mathrm{a}}$ CASPT2 results from ref $^{38}$

${ }^{\mathrm{b}}$ Experimental results from ref ${ }^{38}$

${ }^{\mathrm{c}}$ CASPT2 results from ref ${ }^{12}$

${ }^{\mathrm{d}}$ Experimental result from ref ${ }^{39}$ 
Table 2. Vertical excitation energies (in $\mathrm{eV}$ ) and oscillator strengths (in parentheses) of the pyridinium radical.

\begin{tabular}{ccccc}
\hline & ${ }^{2} \pi^{\#} \pi^{*}\left(\mathrm{~A}^{\prime \prime}\right)$ & ${ }^{2} \pi^{\#} \sigma^{*}\left(\mathrm{~A}^{\prime}\right)^{\mathrm{a}}$ & ${ }^{2} \pi \pi^{\#}\left(\mathrm{~A}^{\prime \prime}\right)$ & ${ }^{2} \pi \pi^{\#}\left(\mathrm{~A}^{\prime}\right)$ \\
\hline $\mathrm{ADC}(2)$ & $2.01(0.001)$ & $2.05(0.000)$ & $5.21(0.000)$ & $5.68(0.023)$ \\
$\mathrm{CC} 2$ & $1.85(0.001)$ & $1.96(0.000)$ & $5.11(0.000)$ & $5.68(0.032)$ \\
$\mathrm{CASSCF}$ & $1.84(0.001)$ & $2.11(0.000)$ & $4.45(0.000)$ & $5.98(0.025)$ \\
CASPT2 & 1.50 & 2.26 & 4.16 & 5.06 \\
\hline
\end{tabular}

${ }^{a}$ calculated with the aug-cc-pVDZ basis set 
Figure Captions

Figure 1. Geometries of ground-state energy minima of the pyridine-water hydrogen-bonded complex in $C_{1}(\mathrm{a}), C_{\mathrm{s}}(\mathrm{b})$, and $C_{\mathrm{s}}^{\prime}$ (c) symmetries, obtained at the MP2 level.

Figure 2. CASSCF natural orbitals involved in the vertical excitations to the lowest ${ }^{1} \pi \pi^{*}$ and ${ }^{1} n \pi^{*}$ states of the pyridine-water complex.

Figure 3. Energy profiles of minimum-energy paths for hydrogen transfer from water to pyridine in $C_{\mathrm{s}}$ symmetry, calculated with the CASSCF (a) and CASPT2 (b) methods. The stars in (a) represent a two-state conical intersection (blue), a saddle point (green), and a three-state conical intersection (red) (see text for details).

Figure 4. Geometries of the ${ }^{1} \pi \pi_{-}{ }^{1} n \pi^{*}$ conical intersection in the Frank-Condon region (a), the saddle point for hydrogen transfer (b) and the ${ }^{1} \pi \pi^{*}{ }_{-}^{1} n \pi^{*}-\mathrm{S}_{0}$ three-state conical intersection in the hydrogen-transfer region (c) of the pyridine-water complex, calculated at the CASSCF level.

Figure 5. Frontier CASSCF natural orbitals of the pyridine-water complex at the hydrogen-transfer saddle point (a, b) and of the ${ }^{1} \pi \pi^{*}(c, d)$ and ${ }^{1} n \pi^{*}$ biradicals (e, f). 
Figure 6. Energy profiles of minimum-energy paths for hydrogen transfer from water to pyridine in $C_{\mathrm{s}}$ symmetry, calculated with the $\mathrm{ADC}(2)$ method.

Figure 7. CASSCF natural orbitals involved in the vertical excitations to the lowest four excited states of the pyridinium radical.

Figure 8. Energy profiles for the photodetachment of the hydrogen atom from the pyridinium radical in $\mathrm{C}_{\mathrm{s}}$ symmetry, calculated with the CASSCF (lines) and CASPT2 (lines with symbols) methods.

Figure 9. Energy profiles for the photodetachment of the hydrogen atom from the pyridinium radical in $\mathrm{C}_{\mathrm{s}}$ symmetry, calculated with the $\mathrm{ADC}(2)$ method. 
Figure 1.

(a)

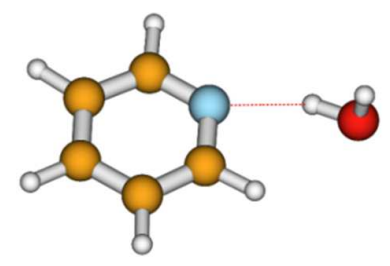

(b)

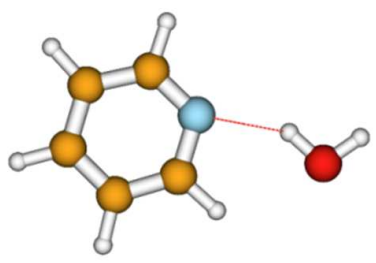

(c)

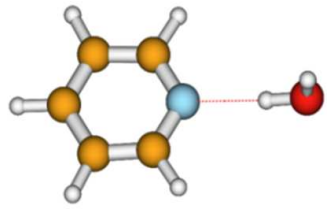


Figure 2.
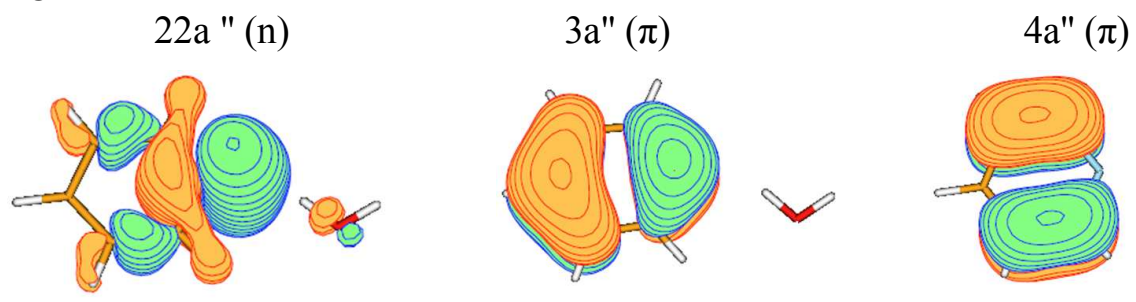

$$
5 \mathrm{a}^{\prime \prime}\left(\pi^{*}\right)
$$

$6 a^{\prime \prime}\left(\pi^{*}\right)$
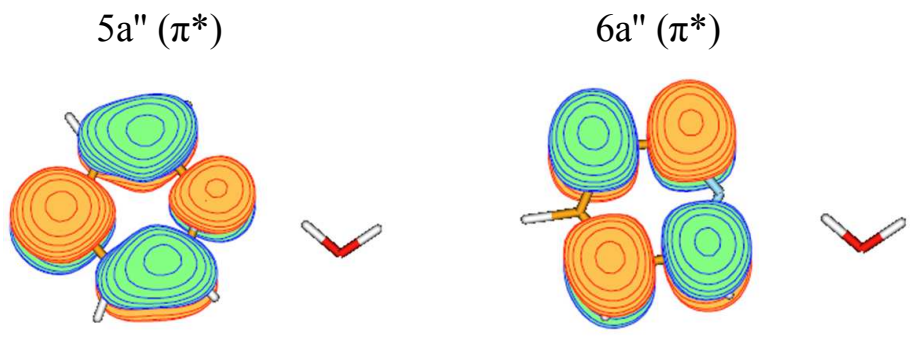
Figure 3.

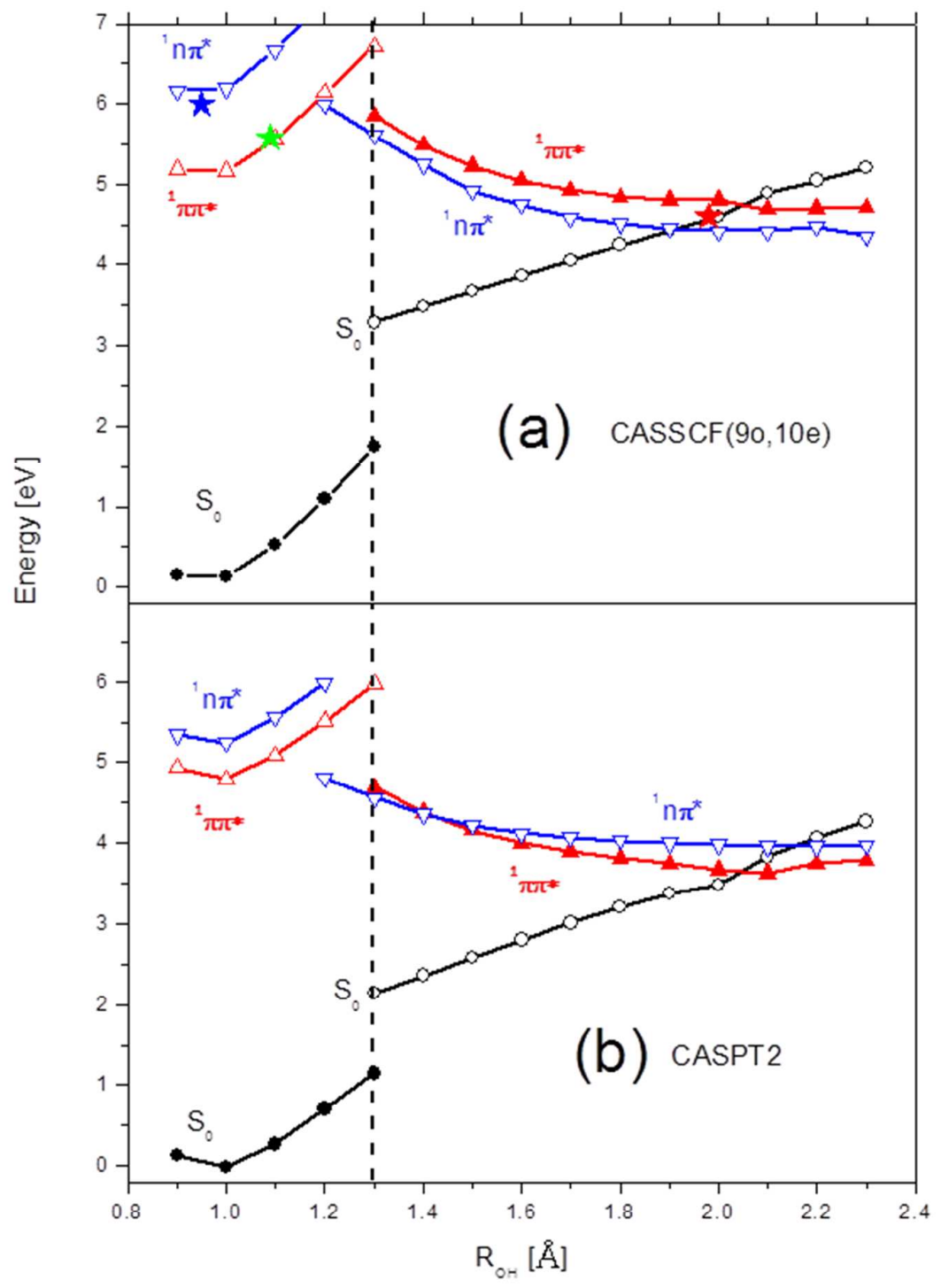


Figure 4.

(a)
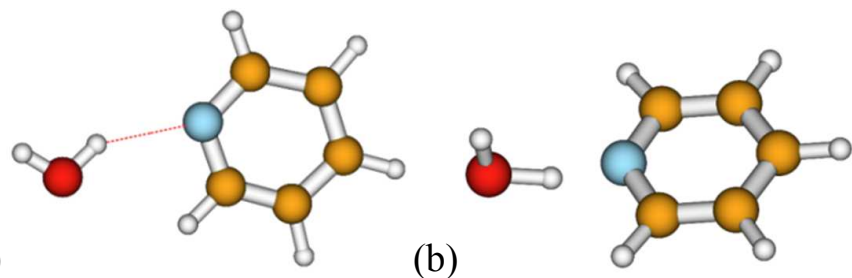

(c)

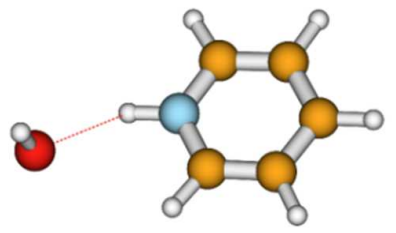


Figure 5.

(a)

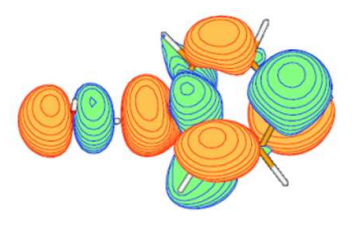

(c)

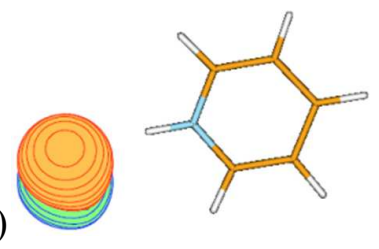

(e)

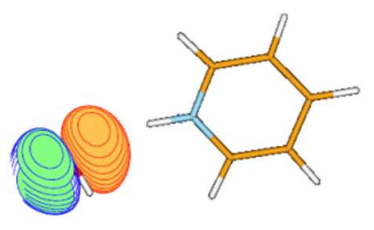

(b)

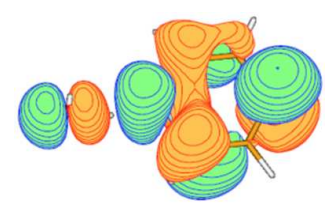

(d)

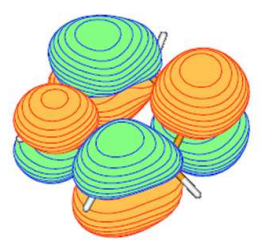

(f)

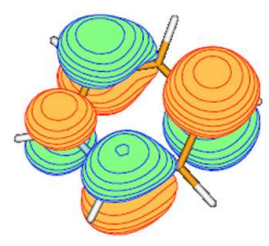


Figure 6.

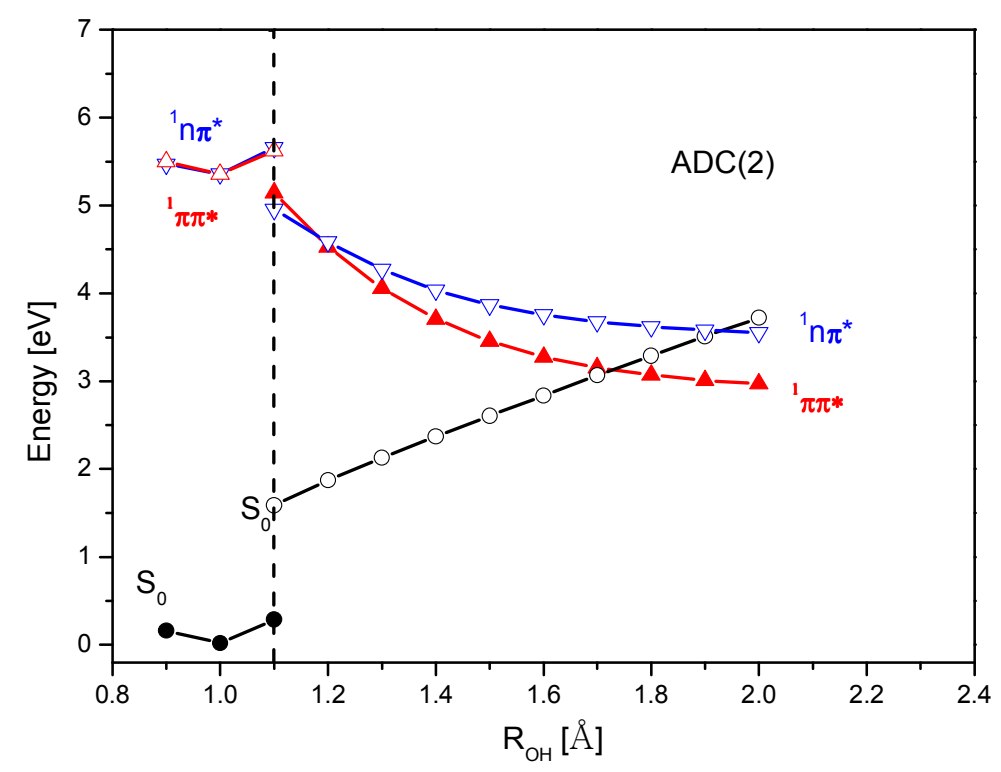


Figure 7.

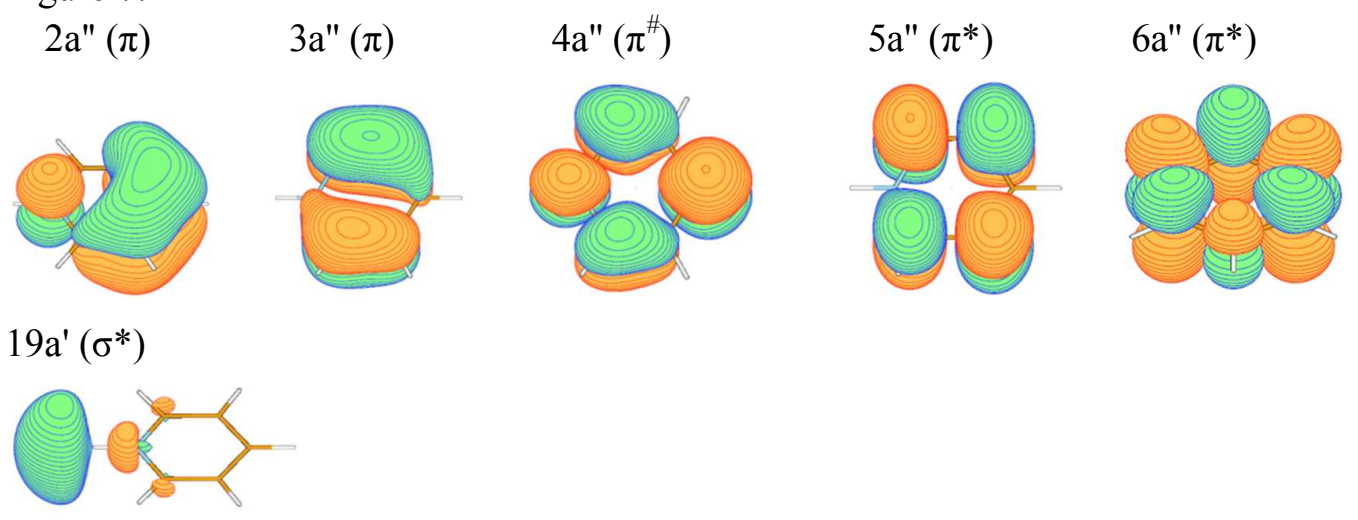


Figure 8.

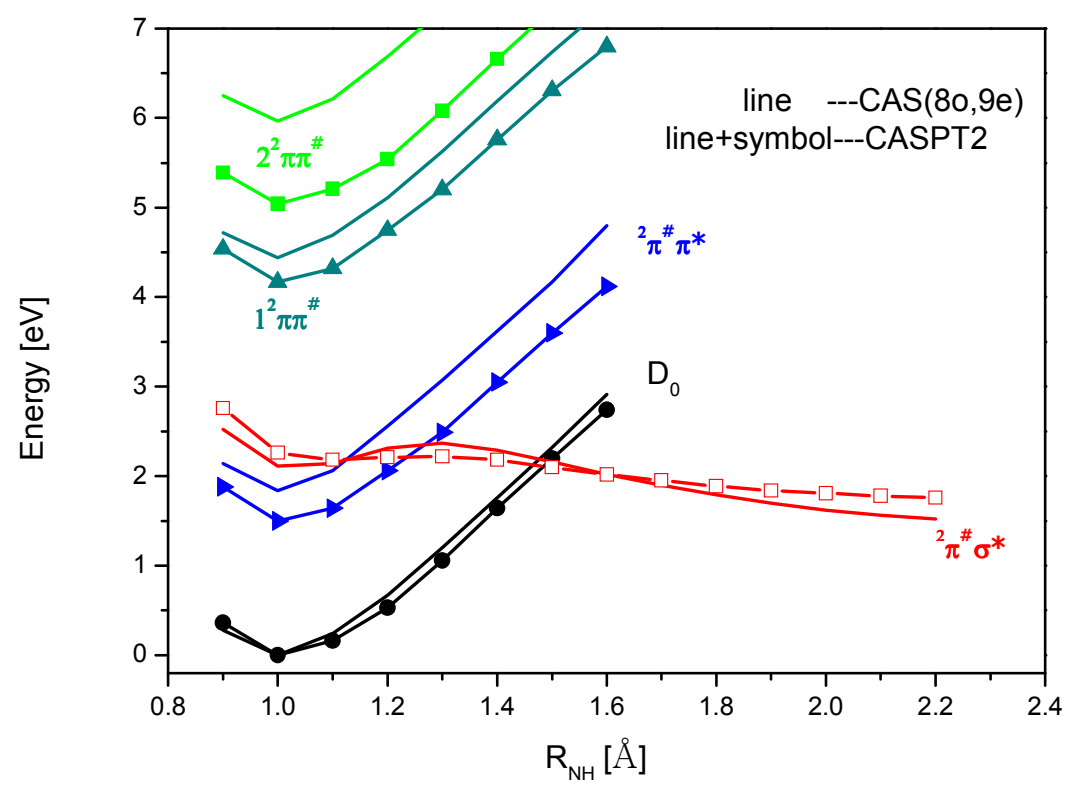


Figure 9.

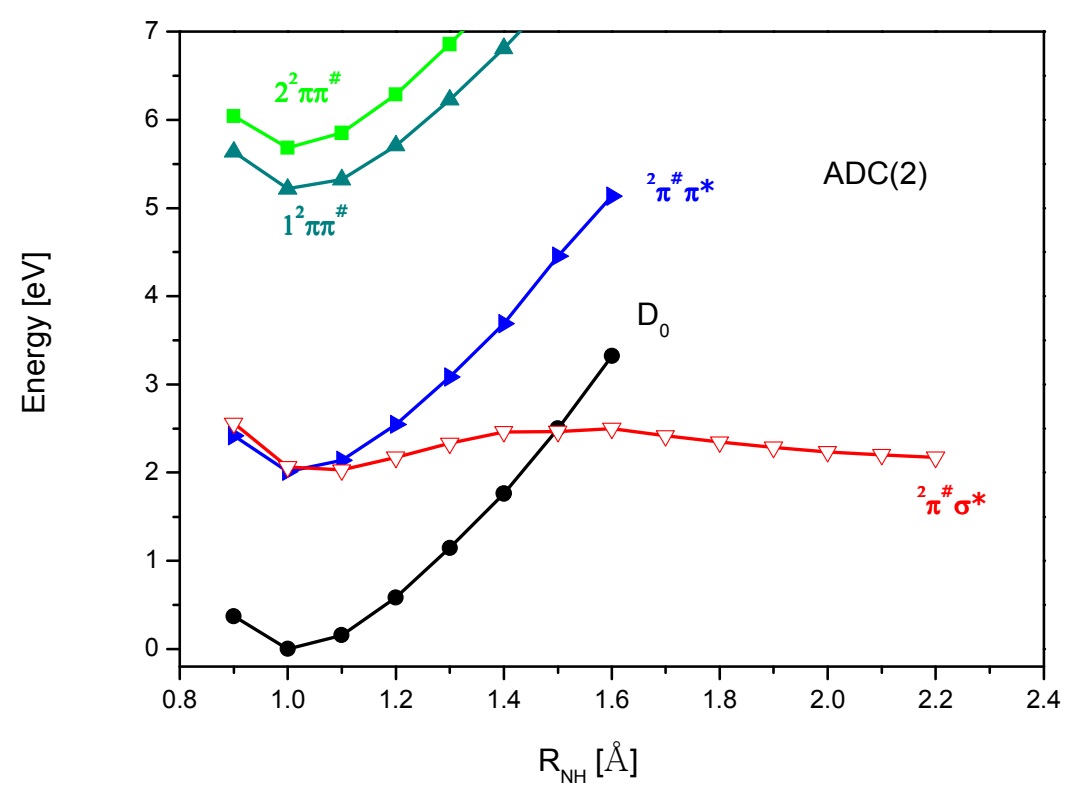




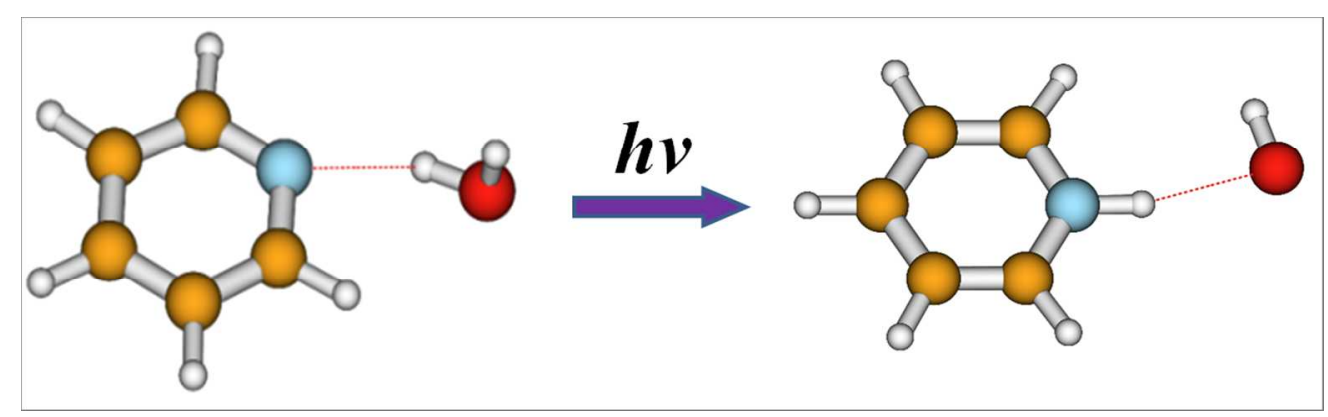

the photoinduced water splitting with pyridine 